\title{
Estimulação cerebral não invasiva e efeito sinérgico do exercício físico sobre a fragilidade e equilíbrio de idosos
}

\author{
Non-invasive brain stimulation and synergistic effect of physical exercise on frailty and \\ balance of elderly
}

Estimulación cerebral no invasiva y efecto sinérgico del ejercicio físico sobre la fragilidad y el equilibrio de los ancianos.

Vernon Furtado da Silva ${ }^{1}$, Eloysa Fonseca Rabaioli', Pedro Henrique Maffini', Caroline Moreno de Azevedo $^{2}$, Maurício Rocha Calomeni', Ivete de Aquino Freire ${ }^{1}$, Luís Felipe Silio ${ }^{1}$, Angeliete Garcez Militão ${ }^{1}$, Rafael Ayres Romanholo ${ }^{4 *}$, João Rafael Valentim-Silva ${ }^{1}$.

\section{RESUMO}

Objetivo: Investigar os efeitos de um programa de intervenção do exercício físico associado à estimulação cerebral não invasiva na fragilidade e equilíbrio de idosos frágeis com declínio do estado mental. Métodos: Quarenta idosos foram selecionados para a pesquisa e posteriormente divididos em dois grupos experimental e controle, $G C(n=20)$ e GE $(n=20)$. A variável fragilidade foi avaliada pela escala de Edmonton e a variável equilíbrio avaliada pelo teste de Tinetti. Os testes foram aplicados em dois momentos, antes e após 60 dias de intervenção. A intervenção consistiu em exercícios físicos para os grupos controle e experimental. Para GE, um procedimento não invasivo de estimulação cerebral (ECNI) foi adicionado aos exercícios físicos e, para o GC, um procedimento de estimulação placebo com ruído branco (PEPL). Cada um desses procedimentos de estimulação durou 20 minutos em cada sessão de intervenção. Resultados: após 60 sessões de estímulos, o GC e o GE mostraram uma diminuição nos níveis de fragilidade, mas apenas o GE melhorou em termos de equilíbrio. Conclusões: Verificou-se que os exercícios físicos são capazes de diminuir a fragilidade física, no entanto, a associação do ECNI aos exercícios físicos foi necessária para melhorar também o equilíbrio dos idosos pesquisados.

Palavras chave: Fragilidade, Exercício físico, Estimulação cerebral não invasiva, Quedas de idosos.

\begin{abstract}
Objective: To investigate the effects of a physical exercise intervention program associated with noninvasive brain stimulation on the fragility and balance of frail elderly with declining mental status. Methods: Forty elderly were selected for the research and later divided into two experimental and control groups, $C G(n=20)$ and $E G(n=20)$. The frailty variable was evaluated by the Edmonton scale and the balance variable evaluated by the Tinetti test. The tests were applied at two times, before and after 60 days of intervention. The intervention consisted of physical exercises for the control and experimental groups. For GE, a non-invasive brain stimulation procedure (ECNI) was added to physical exercises, and for $C G$ a white noise placebo stimulation procedure (PEPL). Each of these stimulation procedures lasted 20 minutes in each intervention session. Results: after 60 stimulus sessions, both the $C G$ and the EG showed a decrease in the levels of fragility but
\end{abstract}

\footnotetext{
${ }_{1}$ Universidade Federal de Rondônia (DEF/UNIR), Porto Velho - RO.

2 Universidade Estácio de Sá, Cabo Frio - RJ.

3 Institutos Superiores de Ensino do Censa, Goytacazes - RJ.

${ }^{4}$ Instituto Federal de Educação, Ciência e Tecnologia de Rondônia (IFRO), Cacoal - RO.

*E-mail: rafael.ayres@ifro.edu.br
} 
only the GE improved in terms of balance. Conclusions: It was found that physical exercises are capable to diminish physical frailty, however, the association of ECNI to physical exercises was needed to also improve balance of the elderly under the research.

Key words: Frailty, Physical exercise, Non-invasive brain stimulation, Fall and the elderly.

\section{RESUMEN}

Objetivo: Investigar los efectos de un programa de intervención de ejercicio físico asociado con la estimulación cerebral no invasiva sobre la fragilidad y el equilibrio de ancianos frágiles con estado mental en declive. Métodos: Cuarenta personas mayores fueron seleccionadas para la investigación y luego divididas en dos grupos experimentales y de control, $C G(n=20)$ y $E G(n=20)$. La variable de fragilidad fue evaluada por la escala de Edmonton y la variable de equilibrio evaluada por la prueba de Tinetti. Las pruebas se aplicaron dos veces, antes y después de 60 días de intervención. La intervención consistió en ejercicios físicos para el control y grupos experimentales. Para GE, se agregó un procedimiento de estimulación cerebral no invasiva (ECNI) a los ejercicios físicos, y para $C G$ un procedimiento de estimulación con placebo de ruido blanco (PEPL). Cada uno de estos procedimientos de estimulación duró 20 minutos en cada sesión de intervención. Resultados: después de 60 sesiones de estímulo, tanto el CG como el EG mostraron una disminución en los niveles de fragilidad, pero solo el GE mejoró en términos de equilibrio. Conclusiones: se descubrió que los ejercicios físicos son capaces de disminuir la fragilidad física, sin embargo, la asociación de ECNI a los ejercicios físicos era necesaria para mejorar también el equilibrio de los ancianos en la investigación.

Palabras clave: Fragilidad, Ejercicio físico, Estimulación cerebral profunda, Caídas de ancianos.

\section{INTRODUÇÃO}

Fragilidade física em idosos tem sido descrita na literatura associada à uma condição sindromática caracterizada por diminuição em força, resistência geral, baixa função fisiológica, além de outras comorbidades. Embora não possa ser prevista como um mal estritamente relacionado a estágios idosos da vida, a mesma tende a drasticamente afetar a vida de muitas pessoas acima de 65 anos de idade, inclusive, sendo correntemente associada ao aumento da propensão de quedas e outros várioas acidentes (LENARDT $\mathrm{MH}$, et al., 2016).

A Associação Médica Americana (American Medical Association) aponta para uma estimativa de que entre 10 a $25 \%$ de indivíduos acima de 65 anos apresentam algum grau de fragilidade e, se acima de 80 anos, este índice sobe para $40 \%$. Um agravante que deve ser considerado demonstra que pode haver uma associação entre a fragilidade com o risco de morte, como apontado em referência a população japonesa (FRIED LP, et al., 2004).

Apesar de não serem muitos os itens mensuráveis inerentes à fragilidade física humana como históricos de quedas, capacidade funcional e qualidade de vida, os desfechos são multivariados, incluindo possíveis agravamentos de doenças incapacitantes instaladas, aumento da possibilidade de quedas, morbidades diversas, baixa autoestima, incapacidade física, aumento da hospitalização e da possibilidade de morte. Estes efeitos deletérios da fragilidade física não têm causa nela somente, mas também em uma associação de fatores físicos e mentais que atinge parte dos idosos, aumentando a sua incidência conforme a idade (CURCIO CL e VARFAEI A, 2019). De fato, estudos passados e recentes têm evidenciado uma relação entre funções cognitivas e fragilidade física permitindo-se à hipótese que esta relação tem alguma conexão com mudanças estruturais e funcionais que ocorrem no corpo e no cérebro ao longo do processo de envelhecimento (CURCIO CL e VARFAEI A, 2019; ROBERTSON DA, et al., 2013).

Devido ao fato de ser, a fragilidade física, uma condição que significativamente afeta a vida de idosos, urge a necessidade de buscar-se ferramentas eficazes para a prevenção e terapêutica dessa condição. Possivelmente a relação entre condições físicas de indivíduos frágeis associadas a perturbações funcionais cognitivas, podem, quando tratadas, revelar efeitos benéficos. 
Apesar desta possível relação que vem sendo estabelecida na literatura a maioria das pesquisas sobre esta temática tem abordado a perspectiva de ser a intervenção física específica a melhor forma de prevenção de estados físicos frágeis, bem como para a qualidade de vida de indivíduos apresentando fragilidade. Contudo, intervenções que ajudem a propiciar, mais diretamente, ao cérebro, condições propícias à estruturação e controle das múltiplas funções orgânico físicas ainda carecem de mais investigações (SILVA V, et al., 2017; FRIED LP, et al., 2004).

Em função do anteriormente exposto, no presente estudo, um procedimento de intervenção baseado em estimulação cerebral não invasiva associado a um treinamento de ordem cognitiva e física foi aplicado a idosos frágeis, levando-se em conta evidências da literatura que apontam a estimulação cerebral não invasiva na modalidade de biofeedback como um instrumento que pode beneficiar funções cognitivas em idosos saudáveis e/ou com decréscimos da função cognitiva (CALOMENI MR, et al., 2017).

Dessa forma consubstanciando a probabilidade teórica de um programa composto por exercícios físicos e mentais, conjugando a uma técnica de estimulação cerebral não invasiva pudesse beneficiar a performance motriz e cognitiva de idosos frágeis a ele submetidos e diminuído níveis iniciais de equilíbrio e da fragilidade em si. Existem características que são mensuráveis como por exemplo a força muscular e equilíbrio corporal que buscam identificar a síndrome da fragilidade em idosos, com isso é de suma importância avaliar os efeitos de programa que estimulam essas variáveis em populações idosas, que estão mais suscetíveis a declínios dessas valências físicas e psíquicas.

Por fim, o objetivo do estudo foi investigar os efeitos de um programa de intervenção com exercícios físicos associados à estimulação cerebral não invasiva sobre a fragilidade e probabilidade de queda em idosos frágeis com declínio do estado mental.

\section{MÉTODOS}

O presente estudo é do tipo quase experimental uma vez que possuiu grupo controle, grupo experimental, mas a seleção inicial dos voluntários foi realizada através de amostragem não randomizada (por conveniência). Diante da sua submissão para execução, foi aprovado por um Conselho de Ética ligado ao Conselho Nacional de Saúde (CNS) e segue todas as orientações da lei 466 de 2012 que autoriza a pesquisa com seres humanos, recebendo o número de CAAE 67198217.0.0000.5524.

A amostra para o estudo se originou de um grupo composto por 340 sujeitos idosos com idade $=$ ou $>$ que 65 anos, atendidos em dois centros de convivência em Porto Velho - RO. Após a observância rígida dos critérios de inclusão/exclusão e aplicação do teste identificador de fragilidade, apenas 49 dos voluntários permaneceram no estudo.

Os critérios que orientaram a inclusão foram: (a) idade acima de 65 e até 80 anos, (b) escolaridade entre qualquer posição no nível médio e superior, (c) obtenção de escores que pudessem superar a pontuação da linha de corte dos testes de base (baseline), (d) concordarem e assinarem de próprio punho e/ou via responsável legítimo, o termo de consentimento livre e esclarecido. Não poderia participar da pesquisa qualquer dos voluntários que, (a) apresentasse dificuldades da fala, visão e/ou audição que evidenciasse desequilíbrio em qualquer tipo de função sensorial, (b) condições físicas que impedissem de realizar os testes, (c) transtorno cognitivo que inviabilizasse o entender das questões dos testes, (d) escore menor ou igual 10 no teste MEEM e (e) não concordassem com a assinatura do termo de consentimento livre e esclarecido.

Assim foram formados dois grupos de idosos identificados anteriormente como possuidores de fragilidade em nível moderado. Eles compuseram dois grupos aleatoriamente divididos de forma que cada grupo contivesse um mesmo número de homens e mulheres. Um dos grupos foi denominado controle com fragilidade moderada ( $G C n=20$ ) e o outro experimental com fragilidade moderada ( $G E n=20$ ).

Para se determinar o estado mental comparativo entre os dois grupos, se fez uso do Mini Exame de Estado Mental (MEEM), sendo que apenas como fator diagnóstico em referência ao processo de inclusão/exclusão. Os testes utilizados na verificação dos dados de base e pós-intervenção dos dois grupos foram a Escala de 
Fragilidade de Edmonton validada para pesquisas no Brasil Fabrício-Wehbe FSCC, et al., (2009); Escala de mobilidade e equilíbrio de Tinetti Province MA et al., (1995) e; Mini exame de estado mental (MEEM) aplicado de acordo com as orientações do próprio teste (JOLLY M, et al., 2014).

Edmonton Frail Scale (EFS) Esta escala avalia 9 domínios, incluindo duas avaliações baseadas no desempenho: o Clock Drawing Test para cognição e o "Timed Up and Go" para equilíbrio e mobilidade. Os outros domínios são humor, independência funcional, uso de drogas, apoio social, nutrição, atitudes em relação à saúde, continência, doenças médicas e qualidade de vida. A pontuação máxima possível é de 17 pontos, o que aponta para um alto nível de fragilidade. Uma pontuação de 0 a 4 indica falta de fragilidade, 56 vulnerabilidade aparente, 7-8 fragilidade leve, 9-10 fragilidade moderada e 11 fragilidade grave.

Teste de Tinetti constituído por 16 itens: 9 para equilíbrio e 7 para marcha. O teste classifica características como velocidade da marcha, passo, simetria e equilíbrio enquanto o indivíduo em teste está parado no lugar e enquanto gira com os olhos fechados. A pontuação para cada exercício varia de 0 a 1 ou 0 a 2, com uma pontuação mais baixa indicando pior capacidade física. A pontuação geral é a soma da pontuação do equilíbrio corporal e da marcha. A pontuação máxima é 12 na marcha, 16 no equilíbrio corporal e 28 na pontuação geral. Uma pontuação <19 pontos refere-se a um alto risco de queda, entre 19 e 24 pontos, um risco moderado de queda e acima de 24 pontos, um baixo risco de queda.

O MEEM teve sua validação no Brasil e seu conteúdo é dividido em sete categorias. Cada categoria tem como objetivo avaliar funções cognitivas específicas, e os resultados variam de zero a 30 pontos, onde pontuações mais altas representam melhores funções cognitivas. Considerando a influência do fator nível de escolaridade, os escores 18 (nível médio de escolaridade) e 26 (alto nível de escolaridade) são normalmente definidos como pontos de corte para determinar a classificação de um indivíduo no teste (WEHBE FSCC, et al., 2009).

\section{Procedimento de intervenção}

Os grupos controle (GC) e o experimental (GE) praticaram o PEFM, enquanto o GE recebeu, ainda, o procedimento NIBS e o GC, um procedimento tipo o NIBS (PEPL), mas com sinais placebos (ruído branco). Para o NIBS, um sintetizador de sinais auditivos emitiu o estímulo a uma frequência de 8 a $12 \mathrm{hertz}(\mathrm{Hz}) \mathrm{na}$ configuração de emissão por binaural beats com uso de um sintetizador produzido pela Touch TechnologyCanadá e de ampla aplicação em estudos nesta linha de investigação (DA SILVA RA, et al., 2016; CALOMENI MR, et al., 2017).

Após as correspondentes intervenções os grupos praticaram o PEFM cuja parte principal foi composta por exercícios de força e resistência dos membros inferiores, abdômen, membros superiores, equilíbrio e coordenação motora geral, todos sob forma de circuitos. Os exercícios mentais foram inseridos entremeados aos exercícios físicos. Durante cada circuito, havia a possibilidade de uma dupla escolha, fazendo com que os idosos mantivessem bom estado de atenção e a vitalidade mental à tomada da decisão certa e de acordo com o estímulo que fora dado.

O nível de intensidade na execução dos exercícios foi determinado pela autopercepção do esforço despendido dos executantes. Dessa forma, quando qualquer um dos idosos se cansava ou sentia-se em desconforto, ele/ela podia fazer uma pausa e descansar. Além disto, foram monitorados por meio do controle da frequência cardíaca. Para esta tarefa foi utilizado o monitor cardíaco Polar modelo FTI. A determinação da frequência cardíaca ideal de treinamento foi realizada por meio da Frequência cardíaca de reserva. A equação para se calcular a faixa ideal de treinamento foi como descrita a seguir.

Frequência cardíaca alvo $=(($ FCmáx - FCrep $)$ * percentual da intensidade $)+$ FCrep Adicionalmente, utilizou-se a escala de esforço auto percebido de Borg, adaptada para o Brasil, definindo que o esforço auto percebido tinha que ser considerado tolerável durante toda a intervenção pelos exercícios físicos e, portanto, sendo classificada entre 6 e 7 de esforço (BORG GAV, 1982). Para se determinar a percepção de esforço seguiu-se o seguinte nível de intensidade relacionada à condição de esforço e, face à frequência cardíaca. 
Tabela1 - Escala subjetiva de esforço.

\begin{tabular}{lc}
\hline Esforço percebido & Frequência Cardíaca (batimentos por minuto) \\
\hline 0 (repouso) & $60-80$ \\
1- muito leve & 90 \\
2 - muito leve & 100 \\
3 - muito leve & 110 \\
4 - leve & 115 \\
5 - leve & 120 \\
6 - moderado & 130 \\
7 - moderado & 140 \\
8 - intenso & 160 \\
9 - muito intenso & 180 \\
10 - exaustivo & 200 \\
\hline
\end{tabular}

Fonte: Borg GAV (1982)

Os participantes, receberam exercícios de prévia habituação, que consistiu em instrução seguida por 5 sessões de exercícios para se adaptarem à sensação de intensidade moderada. Em seu total, o programa de intervenção foi de 60 minutos por sessão, sendo 20 minutos para a aplicação do NIBS/PEPL, 5 minutos para o aquecimento, 30 minutos para os exercícios físicos e 5 minutos para desaquecimento. Cada sessão foi desenvolvida às segundas, quartas e sextas feiras

\section{Procedimentos estatísticos}

Utilizou-se o teste de Komolgorov-Smirnov para se determinar a normalidade dos dados dos grupos, o teste de Mann-Whitney nas comparações intragrupos dos escores pré e pós-intervenção, a correlação de Spearman para se verificar associações entre as variáveis em estudo e, o teste de Cohen's $D$, à identificação da magnitude das relações entre elas. Todas as comparações e correlações foram executados em referência a $5 \%$ de probabilidade de erro por aceitação e ou rejeição das hipóteses inerentes à pesquisa.

\section{RESULTADOS}

A Tabela 2 demonstra as características do grupo voluntário e o teste de Mann-Whitney com significância de $5 \%$ foi utilizado para se determinar a diferença entre os grupos.

Tabela 1 - Dados descritivos das características dos dois grupos e valor de $p$ do teste estatístico entre os grupos

\begin{tabular}{ccccccccc}
\hline Grupos & Idade & $\begin{array}{c}\text { Valor de } \\
\boldsymbol{p}\end{array}$ & Edmonton & $\begin{array}{c}\text { Valor de } \\
\boldsymbol{p}\end{array}$ & Tinetti & $\begin{array}{c}\text { Valor de } \\
\boldsymbol{p}\end{array}$ & MEEM & $\begin{array}{c}\text { Valor de } \\
\boldsymbol{p}\end{array}$ \\
\hline $\mathrm{GC}$ & $73,3 \pm 4,5$ & 0,528 & $10 \pm 1,1$ & 0,516 & $15 \pm 2,8$ & 0,0795 & $24 \pm 5,4$ & \\
GE & $73,9 \pm 3,8$ & & $10 \pm 1,6$ & & $16 \pm 3,3$ & & $24 \pm 2,3$ & 0,293 \\
\hline
\end{tabular}

Fonte: Silva V.F et al., 2019.

Na Tabela 1, pode-se observar que tanto no fator idade, quanto nos fatores intrínsecos à fragilidade e a propensão à queda, antes da intervenção os grupos GC e GE não apresentaram diferenças entre eles. Ou seja, para todas essas variáveis o fator de determinação de diferença estatística não foi significativo ( $p>0.05$ para todas as comparações). 
$\mathrm{Na}$ Figura 1 estão expostos os dados resultantes da experimentação realizada mostrando as comparações entre os dados da performance dos grupos nos testes de Fragilidade e Risco de Quedas em momentos pré-intervenção e pós-intervenção.

Figura 1 (A e B) - Quarenta idosos diagnosticados com fragilidade moderada
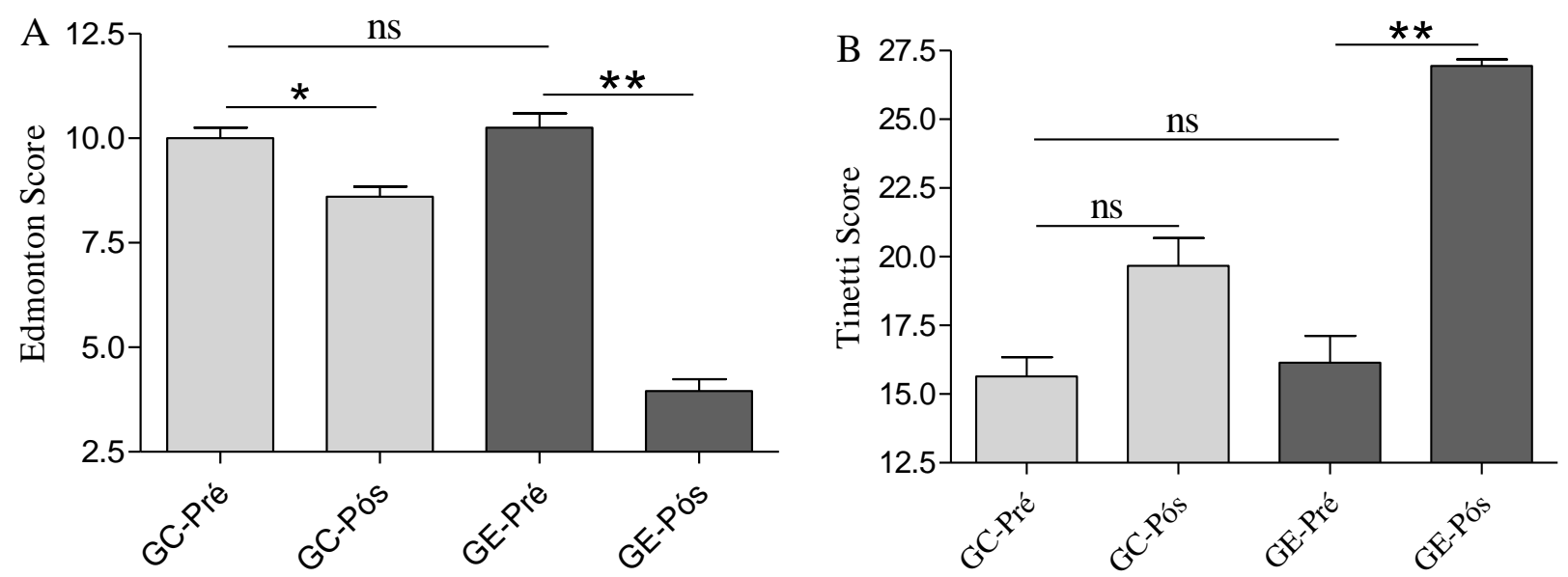

Legenda: foram divididos em GC $(n=20)$ e $G E(n=20)$ e submetidos a um programa de intervenção composto por exercícios físicos para ambos os grupos, todavia, para o GE foi adicionada estimulação cerebral não invasiva (ECNI) real e para o GC, estimulação placebo com ruído branco (PEPL) por 20 minutos cada sessão. $\mathrm{Ns}=$ não significativa, ${ }^{*}=$ sig. $0,01,{ }^{* *}=$ Sig. 0,001 .

Fonte: Silva V.F et al., 2019.

\section{Exercícios físicos de intensidade moderada conjugados à estimulação cerebral não invasiva por emissão binaural, fez reduzir o nível de fragilidade e de propensão à queda de idosos frágeis.}

A avaliação dos níveis de fragilidade por meio da Escala de Edmonton evidenciou que o GC obteve escore de $10 \pm 1,1$ no pré-teste e nos pós teste $8,6 \pm 1,1$ e apresentando diferença entre os dois momentos $(p=0,025)$ (Fig. 1A). Ainda em relação à fragilidade o GE obteve escore de $10 \pm 1,6$ no pré-teste e no pós-teste de $4 \pm 1,3$ com diferença entre os dois momentos $(p=0,0009)$ (Fig. $1 \mathrm{~A})$. Em relação tamanho do efeito o GC obteve resultado médio $(d=0,69)$ enquanto GE grande $(d=0,88)$.

\section{Exercícios físicos de intensidade moderada, fez reduzir o nível de fragilidade, mas não o de propensão à queda de idosos frágeis.}

A avaliação da propensão de quedas por meio do teste de Tinetti demonstrou que o GC obteve escore de $15 \pm 2,8$ no pré-teste e no pós-teste de $20 \pm 4,7$ sem diferença entre os momentos ( $p=0.051)$ (Fig. $1 \mathrm{~B}$ ). O GE obteve escore de $16 \pm 3,3$ no pré-teste e no pós-teste de $27 \pm 1,1$ com diferença entre os dos momentos $(p=0,0016)$ (Fig. 1B).

\section{DISCUSSÃO}

A literatura atual mostra uma estreita relação entre a fragilidade e uma série de mudanças de base fisiológica que se refletem negativamente no comportamento motor, cognitivo, afetivo e social da significativa maioria das pessoas idosas que a contrai. Essas se refletem principalmente em redução da homeostase orgânica como um todo e, consequente perda da autonomia e independência funcional (BORTZ WM II, 2002). A sua alta prevalência em qualquer sociedade recomenda a busca de meios alternativos que atendam ao seu tratamento e sua prevenção. Em participação à essa linha de pensamento, no presente estudo investigamos 
os efeitos da aplicação de um programa de exercícios físicos e mentais (PEFM) combinado, ou não, à uma técnica de estimulação cerebral não invasiva (NIBS), em dois grupos de idosos com fragilidade moderada e nível alto de tendência à queda.

Os resultados mostram que tanto a conjugação do PEFM ao procedimento NIBS, quanto a aplicação do PEFM isolado significativamente beneficiaram os dois grupos de idosos em termos de redução no nível de fragilidade por eles apresentados antes do processo de intervenção. Mesmo assim, os dados da estatística intergrupo, bem como a plotagem destes na Figura (1-A) indicam um possível maior efeito da intervenção conjugada (PEFM + NIBS) comparativamente à que foi aplicada isoladamente (PEFM), todavia sem correspondência significativa.

Esses resultados mostrando os efeitos benéficos do exercício sobre o fator fragilidade são bem comuns na literatura nessa linha. Várias pesquisas têm revelado resultados similares, mesmo quando da aplicação de exercícios em intensidade e composição diferentes à praticada nesta pesquisa. Algumas evidências mostram que idosos frágeis podem ser mais beneficiados tanto por treinamento com base em exercícios resistidos e de equilíbrio do que os de natureza aeróbia. Além disto, um considerável número de autores examinando os efeitos treinamento com base em multicomponentes de exercícios aconselham o uso desta prática sobre as demais, principalmente em função de prover respostas terapêuticas mais amplas à variedade de disfunções cognitivas e biofísicas que idosos frágeis tendem a apresentar (NG TP, et al., 2015).

Diferentemente dos resultados que as duas intervenções mediadas por exercícios físicos produziram em relação ao fator fragilidade, em termos da variável tendência a queda o programa de exercícios físicos e mentais (PEFM) por si só, não produziu mudanças no índice de tendência à queda que os idosos do GC apresentaram em testes pré-intervenção. Como se pode verificar na plotagem dos dados e resultados estatístico das comparações intragrupo na Figura (1-B), apenas o GE revelou significativos ganhos por efeito da intervenção que combinou os procedimentos PEFM + NIBS.

Em princípio o baixo efeito do PEFM mostrado em relação à performance do GC no teste de Tinetti é bastante contraditório, considerando-se que em virtude dos custos alarmantes de tratamentos relacionados a queda de idosos, um grande número de programas terapêuticos, incluindo exercícios físicos aplicados isoladamente e / ou combinados têm sido utilizados com sucesso tanto na minimização quanto na prevenção de agravos que a queda produz sobre a saúde geral de idosos (BURNS ERB, et al., 2016; SILVA V, et al., 2017; FRANCO MR, 2014; GILLESPIE LD, 2012).

Todavia, apesar da grande perspectiva que se possa ter em termos da relação benéfica que a literatura mostra entre programas de exercícios, prevenção e minimização de problemas associados ao envelhecimento humano, nem todas as intervenções utilizando programas de exercícios neste sentido são capazes de produzir bons resultados. Ou seja, além da característica da composição da série de exercícios, a intensidade ou dosagem na aplicação de um programa pode fazer uma grande diferença. E muito embora não se tenha ainda bem definido os melhores tipos e níveis de intensidade em benefício de idosos, alguns autores recomendam os exercícios resistidos como sendo bons, enquanto outros, justificam que idosos não respondem bem e tendem evitar aderência a exercícios de baixa intensidade (ABE D e FUKUOKA HM, 2019), (JOHNSON SL, et al., 2019).

No programa de exercícios aqui utilizado, buscou-se uma aproximação da aplicação de intensidade moderada, mas com observações de feedback que o fazia aproximar-se do nível de baixa intensidade. De uma certa forma, alguns autores em pesquisas com idosos aconselham a sempre começar de um nível de baixa intensidade em graduados avanços, mesmo quando de uso de metodologia com carga de alta intensidade. Isto ajudando com a familiarização dos exercícios, prevenindo contra lesões além de outros benefícios à adaptação como um todo (IZQUIERDO M e CADORE E, 2014).

Os pressupostos orientadores da lógica de se contrapor elementos de estimulação orgânica (NIBS e PEFM) à resultantes psicofísicas emergentes dos testes de Tinneti e da Escala de Fragilidade de Edmonton são oriundos de evidências científicas quanto a existência de interatividade entre eles. Ou seja, a prática de exercícios físicos de forma regular ou em programas de curto prazo pode resultar em base de prevenção 
contra o avanço de algumas doenças que muitas das vezes se alinham ao processo de envelhecimento humano (CADORE EL, et al., 2013; PAILLARD T, et al., 2019). Além disso, recompor funções cognitivas, biofísicas e psíquicas minimizando riscos demenciais e/ou degenerações que fragilizam o comportamento de uma grande maioria de idosos (CRUSH EA e LOPRINZI PD, 2017; DUZEL E, et al., 2016; PAILLARD T, et al., 2019).

Em um outro viés, mas em função relacionada, comumente a aplicação da NIBS com enfoque em cognição, memória e percepção resultam em melhora de equilíbrio hemisférico e equalização da atividade cerebral, com efeitos relacionados a melhorias em capacidade funcional e predisposição do cérebro à manutenção de foco, atenção e competência de processamento mental (CALOMENI MR, et al., 2008; O'CONNELL NE, et al., 2014; HALLETT M, 2007).

Tendo-se como base uma analogia dos pressupostos acima enfatizados, os resultados desta pesquisa se mostram a eles aderentes, compreendendo-se que a aplicação do PEFM teve repercussões benéficas sobre o nível de fragilidade e de tendência a queda nos dois grupos de idosos, embora que, em termos de analogia estatística, tais efeitos só possam ser atribuídos a sua relação com o fator fragilidade. A observância da Figura (1-B), deixa claro que o PEFM teve repercussões benéficas também sobre a variável probabilidade de queda, embora em montante não suficiente para se justificar um valor de significância estatística. Nesse caso, a aplicação da NIBS revela-se sob forma de efeito aditivo que merece destacada atenção, na interpretação dos efeitos do PEFM tanto em relação à variável fragilidade (Figura 1-A) quanto em referência à variável tendência à queda (Figura 1-B). Isto é, a aplicação do PEFM teve efeito significativo na performance do GC em referência a variável fragilidade e adicional à variável tendência à queda, enquanto que a intervenção combinando o PEFM e a NIBS, pode ter influenciado a performance do GE, de forma inversa.

\section{CONCLUSÃO}

Para concluir, o programa de treinamento combinando exercícios físicos e mentais com o procedimento de estimulação cerebral por emissão binaural promoveu a minimização no nível de fragilidade e de tendência a queda nos idosos aqui pesquisados. Além disto, quando aplicado sem o procedimento de estimulação, promoveu a minimização da fragilidade, mas não o nível de tendência à queda desses mesmos idosos. Estes resultados se somam aos anteriores mostrando os benefícios do exercício físico sobre uma gama de disfunções biofísicas em pessoas idosas de uma forma em geral. Além disto recomendam a atenção de profissionais na área da saúde para estudos mais aprofundados sobre o uso da estimulação cerebral não invasiva em terapias cognitivas que possam ser aplicadas no tratamento da síndrome da fragilidade e de outros males que infelizmente interagem com o processo de envelhecimento humano.

\section{AGRADECIMENTOS}

Os autores deste trabalho agradecem à FUNDAÇÃO DE AMPARO À PESQUISA DE RONDÔNIA (FAPERO) que por meio da CAPES e propiciou a possibilidade de desenvolvimento deste projeto, ao CNPq/PROPESQ por bolsa de IC, ao Serviço Social do Comercio (SESC) e aos voluntários que participaram de maneira graciosa.

\section{REFERÊNCIAS}

1. $\mathrm{ABE}$ DF, et al. On the simple calculation of walking efficiency without kinematic information for its convenient use. Journal of Physiological Anthropology, 201938 (1):1-6.

2. BORG GAV. Bases psicofísicas de esforço percebido. Med sci esportes exerc, 1982, 14 (5): 377-381.

3. BORTZ WM. Um quadro conceitual de fragilidade: uma revisão. The Journals of Gerontology Série A: Ciências Biológicas e Ciências Médicas, 2002, 57 (5): 283-288.

4. BURNS ER, STEVENS JA, LEE R. Os custos diretos de mortes fatais e não fatais caem entre adultos mais velhos Estados Unidos. Journal of safety research, 2016, 58: 99-103.

5. CADORE EL, et al. Effects of Different Exercise Interventions on Risk of Falls, Gait Ability, and Balance in Physically Frail Older Adults: A Systematic Review. Rejuvenation Res. 2013;16(2):105-114. 
6. CALOMENI MR, et al. Modulatory Effect of Association of Brain Stimulation by Light and Binaural Beats in Specific Brain Waves. Clin Pract Epidemiol Ment Heal. 2017;13(1):134-144.

7. CAMÕES $M$, et al. Exercício físico e qualidade de vida em idosos: diferentes contextos sociocomportamentais. Motricidade, 2016, 12(1): 96-105.

8. CESARI M, et al. A physical activity intervention to treat the frailty syndrome in older persons: results from the LIFEP study. J Gerontol A Biol Sci Med Sci. 2015;70(2):216-22.

9. CRUSH EA, LOPRINZI PD. Dose-response effects of exercise duration and recovery on cognitive functioning. Perceptual and Motor Skills, 2017, 124 (6): 1164-1193.

10. CURCIO CL, et al. A regression tree for identifying risk factors for fear of falling: the International Mobility in Aging Study (IMIAS). The Journals of Gerontology: Series A, 2020, 75 (1): 181-188.

11. DA SILVA RA, et al. Ginástica geral pode melhorar a marcha e a capacidade cardiovascular de idosos. Rev Bras Med do Esporte. 2016;22(4):306-310.

12. DA SILVA, Vernon Furtado, et al. O treinamento físico associado à estimulação cerebral não invasiva modula as ondas corticais, diminuindo a probabilidade de quedas em adultos idosos com fragilidade. Revista Brasileira de Pesquisa e Ciência em Engenharia Avançada, 2019, 6 (5).

13. DUZEL E, PRAAG H, SENDTNER M. Can physical exercise in old age improve memory and hippocampal function? Brain, 2016, 139 (3): 662-673.

14. ELIASOVA I, et al. Non-invasive brain stimulation of the right inferior frontal gyrus may improve attention in early Alzheimer's disease: a pilot study. Journal of the neurological sciences, 2014, 346 (1-2): 318-322.

15. FABRíCIO-WEHBE, SCC, et al. Cross-cultural adaptation and validity of the" Edmonton Frail Scale-EFS" in a Brazilian elderly sample. Revista latino-americana de enfermagem, 2009, 17(6): 1043-1049

16. FRANCO MR, et al. Exercise interventions for preventing falls in older people living in the community. Br $\mathrm{J}$ Sports Med, 2014, 48 (10): 867-868

17. FRIED, LP. et al. Untangling the concepts of disability, frailty, and comorbidity: implications for improved targeting and care. The Journals of Gerontology Series A: Biological Sciences and Medical Sciences, 2004, 59 (3): $255-263$.

18. GARUFFI M, et al: Effects of resistance training on the performance of activities of daily living in patients with Alzheimer's disease. Geriatr Gerontol Int, 2013, 13: 322-328.

19. GILLESPIE LD., et al. Interventions for preventing falls in older people living in the community. Cochrane database of systematic reviews, 2012, 9.

20. HALLETT M. Transcranial magnetic stimulation: a primer. Neuron, 2007, 55 (2): 187-199.

21. IZQUIERDO M, CADORE E. Treinamento da força muscular nos frágeis institucionalizados: uma nova abordagem para combater o declínio funcional e a incapacidade muito tardia da vida. Curr Med Res, 2014, 30 (7): 1385-90.

22. JEFFERIS BJ, et al. Adherence to physical activity guidelines in older adults, using objectively measured physical activity in a population-based study. BMC Public Health, 2014, 14:382.

23. JOHNSON SL. et al. Effect of Lower-Extremity Eccentric Training on Physical Function in Community-Dwelling Older Adults. Physical \& Occupational Therapy In Geriatrics, 2019, 37 (4): 298-312.

24. JOLLY M, et al. Health and quality of life outcomes. Health and quality of life outcomes, 2014, 12 (1): 173.

25. LENARD MH, et al. Frailty and quality of life in elderly primary health care users. Rev Bras Enferm, 2016, 69(3):478483.

26. NG TP, et al. Nutritional, physical, cognitive, and combination interventions and frailty reversal among older adults: a randomized controlled trial. Am J Med. 2015,128(11):1225-36.

27. NOUCHI R, et al. Four weeks of combination exercise training improved executive functions, episodic memory, and processing speed in healthy elderly people: Evidence from a randomized controlled trial. Age (Omaha), 2014, 36(2):787-799.

28. O'CONNELL NE, et al. Non-invasive brain stimulation techniques for chronic pain. Cochrane Database of Systematic Reviews,2014, (4).

29. ÖHMAN H, et al. Effects of exercise on cognition: the Finnish Alzheimer disease exercise trial: a randomized, controlled trial. Journal of the American Geriatrics Society, 2016, 64 (4): 731-738.

30. OKAMURA $\mathrm{H}$, et al. Combined exercise and cognitive training system for dementia patients: a randomized controlled trial. Dementia and geriatric cognitive disorders, 2018, 45 (5-6): 318-325.

31. PAILLARD T, et al.Protective Effects of Physical Exercise in Alzheimer's Disease and Parkinson's Disease: A Narrative Review. J Clin Neurol, 2015, 11(3):212-219.

32. PROVINCE MA, et al. The effects of exercise on falls in elderly patients: a preplanned meta-analysis of the FICSIT trials. Jama,1995, 273 (17): 1341-1347.

33. ROBERTSON DA, et al. Frailty and cognitive impairment-a review of the evidence and causal mechanisms. Ageing research reviews, 2013, 12 (4): 840-851.

34. ROLFSON DB, et al. Validity and reliability of the Edmonton Frail Scale. Age and Ageing. 2006, 35 (5):526-529.

35. SILVA VF, et al. Brain stimulation used as biofeedback in neuronal activation of the temporal lobe area in autistic children. Arquivos de neuro-psiquiatria, 2016, 74 (8): 632-637.

36. SILVA RB, et al. The Effect of Physical Exercise on Frail Older Persons: A Systematic Review. J frailty aging, 2017, 6(2):91-96.

37. SILVA V, et al. Wii-based exercise program to improve physical fitness, motor proficiency and functional mobility in adults with Down syndrome. Journal of Intellectual Disability Research, 2017, 61 (8): 755-765. 
38. SILVA RBB, et al. The Effect of Physical Exercise on Frail Older Persons: A Systematic Review. J frailty aging, 2017,6: 91-96.

39. TAY L, et al. The independent role of inflammation in physical frailty among older adults with mild cognitive impairment and mild-to-moderate Alzheimer's disease. The journal of nutrition, health \& aging, 2016, 20 (3): 288-299.

40. TSAI CL, et al. Effects of acute aerobic exercise on a task-switching protocol and brain-derived neurotrophic factor concentrations in young adults with different levels of cardiorespiratory fitness. Experimental Physiology, 2016,101 (7): 836-850. 